\title{
Riesgo cardiovascular y de diabetes en preescolares
}

\section{Biomarker disorder of cardiovascular and type 2 diabetes in preschoolers in Northwest Mexico: an exploratory study}

Martha Nydia Ballesteros-Vásquez, ${ }_{1}$ Saraí García-Gámez, ${ }^{2}$ Elizabeth Artalejo-Ochoa, ${ }^{2}$ Bianca Vargas-Tequida, ${ }^{3}$ José Antonio Ponce-Martínez, ${ }^{3}$ Alma Elizabeth Robles-Sardin, ${ }^{2}$ María Isabel Grijalva-Haro ${ }^{1}$

\begin{abstract}
Resumen
OBJETIVO: Hacer un análisis exploratorio de las posibles alteraciones en los marcadores clínicos de riesgo cardiovascular y diabetes tipo 2 en preescolares habitantes de la zona urbana y conurbada del municipio de Hermosillo, Sonora, al Noroeste de México.

MATERIALES Y MÉTODOS: Estudio observacional y transversal efectuado en niños de las zonas urbana y conurbada. Se midieron: peso, talla, circunferencia de cintura, porcentaje de grasa corporal y presión arterial. Se evaluaron el perfil de lípidos y las concentraciones de glucosa e insulina.

RESULTADOS: Se estudiaron 82 niños de edad promedio de 5.0 años; 48 de la zona conurbada y 34 de la urbana. Se encontró la coexistencia de problemas de desnutrición, sobrepeso y porcentaje de grasa corporal elevado $(27 \% \pm 0.52)$. En la zona conurbada hubo mayor porcentaje de niños con alteraciones en los lípidos: triglicéridos elevados $(35 \%)$; HDL-c bajo $(25 \%)$, ( $\mathrm{p}<0.01)$, y pre-hipertensión arterial, mientras que en la zona urbana hubo mayor porcentaje de niños con glucosa alterada $(12 \%)$ y concentraciones elevadas de insulina y resistencia a ésta.

CONCLUSIONES: Los hallazgos de este estudio muestran que los factores de riesgo cardiovascular y de diabetes tipo 2 prevalecen en niños de 3 a 6 años habitantes de las zonas urbana y conurbada de Hermosillo, Sonora.

PALABRAS CLAVE: Niños preescolares; malnutrición; lípidos; glucosa; insulina; factores
\end{abstract} de riesgo; enfermedades cardiovasculares; obesidad; diabetes tipo 2; México.

Abstract

INTRODUCTION: Childhood obesity is a health problem that has been growing in the last decades in the world and particularly in Mexico. In addition to lipid alterations, glucose and insulin are risk factors for the development of cardiovascular diseases and diabetes. Studies the Northwest of Mexico have focused on the evaluation of these risk factors in school-age children and have shown the presence of the metabolic syndrome. However, there is insufficient information on cardiovascular health in preschool children.

OBJECTIVE: To perform an exploratory analysis of the presence of possible alterations in clinical biomarkers of cardiovascular risk and type 2 diabetes in preschoolers living in the urban and conurbated zones of Hermosillo, México.

MATERIALS AND METHODS: A cross sectional study was carried out, with two study groups: children living in the urban zone $(\mathrm{ZU})$ and children in the conurbation zone (ZCU). Weight, height, waist circumference, percentage of body fat and blood pressure, lipids, glucose and insulin profile were assessed.

RESULTS: The average age of the participants was 5.0 years $(n=82), 48$ from the ZCU and 34 from the ZU. Problems of malnutrition, overweight and high body fat $(27 \%$ \pm 0.59 ) coexist. No cases of obesity were observed. In the ZCU of the city there is a higher percentage of children with lipid abnormalities: elevated triglycerides (35\%),

\begin{abstract}
${ }^{1}$ Investigadora titular, Departamento de Nutrición y Metabolismo, Coordinación de Nutrición.

${ }^{2}$ Departamento de Nutrición y Metabolismo.

${ }^{3}$ Departamento de Nutrición Pública y Salud.

Centro de Investigación en Alimentación y Desarrollo, AC. Hermosillo, Sonora.
\end{abstract}

Recibido: 1 de diciembre 2017

Aceptado: 2 de julio 2018

Correspondencia

Martha Nydia Ballesteros-Vásquez nydia@ciad.mx

Este artículo debe citarse como Ballesteros-Vásquez MN, García-Gámez $\mathrm{S}$, Artalejo-Ochoa E, Vargas-Tequida B, Ponce-Martínez JA, Robles-Sardin AE, Grijalva-Haro MI. Riesgo cardiovascular y de diabetes en preescolares. Acta Pediatr Mex. 2019;40(1):1-10. 
low HDL-C $(25 \%),(p<0.01)$, and prehypertension, whereas in the ZU there was a higher percentage of children with impaired glucose (12\%) and elevated insulin levels and insulin resistance.

CONCLUSIONS: The findings of this study show the risk factors for type 2 diabetes and that cardiovascular disease are present in children between the ages of 3 and 6 years from ZU and ZCU of the Hermosillo, Mexico.

KEY WORDS: School age children; Malnutrition; Lipid; Glucose; Insulin; Risk factors; Cardiovascular diseases; Obesity; Type 2 diabetes; Mexico.

\section{ANTECEDENTES}

La epidemia de obesidad ha alcanzado a la población infantil de todo el mundo y ha generado la necesidad de evaluar los factores de riesgo de enfermedades no trasmisibles: diabetes tipo 2 y enfermedades cardiovasculares en niños de edad escolar. Hasta ahora, los hallazgos muestran que los escolares tienen alteraciones físicas y metabólicas propias de la obesidad: alteraciones en los lípidos sanguíneos y en los marcadores del metabolismo de la glucosa. ${ }^{1}$ La genética, la dieta y la inactividad física están implicadas en su aparición temprana. ${ }^{2}$

Algunos estudios sugieren que es posible que el exceso de peso ganado antes de los 5 años persista hasta la vida adulta ("obesidad persistente"). ${ }^{3,4}$ A pesar de la obesidad, no es común que las enfermedades cardiovasculares aparezcan durante la niñez o la adolescencia, pero sí es posible observar factores y comportamientos de riesgo que aceleran la aterosclerosis en niños tan pequeños como los de 4 años. ${ }^{5}$

Las concentraciones de lípidos séricos y lipoproteínas aumentan durante la infancia temprana, aproximadamente a los dos años, y alcanzan a las observadas en adultos jóvenes. ${ }^{6,7,8}$ Los estudios epidemiológicos dan cuenta de que si en la niñez se alteran las concentraciones de lípidos sanguíneos, se corre un alto riesgo de sufrir lesiones ateroscleróticas en la edad adulta. ${ }^{9}$ Otras investigaciones establecen que los lípidos sanguíneos en niños se correlacionan con la obesidad y resistencia a la insulina. ${ }^{10}$

Estudios previos de nuestro grupo de trabajo efectuados en población sonorense confirman el sobrepeso y la obesidad en niños de 6 a 9 años, además de señalar el síndrome metabólico en áreas urbanas (7\%) y rurales (5\%). La dieta y la inactividad física son dos de los principales factores implicados. ${ }^{11}$

En México, el problema no parece estar sólo confinado a los niños escolares, pues según la Encuesta Nacional de Salud y Nutrición ${ }^{12}$ $14.1 \%$ de los niños menores de 5 años tienen sobrepeso y obesidad, por lo que es factible que puedan observarse alteraciones en los marcadores de riesgo cardiovascular, como el perfil de lípidos, que sugieren que las enfermedades cardiovasculares se inician a una edad más temprana en esta población.

El objetivo de este estudio fue: hacer un análisis exploratorio de las posibles alteraciones en los marcadores clínicos de riesgo cardiovascular y diabetes tipo 2 en preescolares que viven en la zona urbana y conurbada del municipio de Hermosillo, Sonora, al Noroeste de México. 


\section{MATERIALES Y MÉTODOS}

Estudio observacional y transversal efectuado en niños de las zonas urbana y conurbada de Hermosillo, Sonora. Esta investigación forma parte de la "Evaluación de Seguimiento del Programa de Desayunos Escolares DIF, Sonora" que se lleva a cabo cada año en el Centro de Investigación en Alimentación y Desarrollo, AC (CIAD, AC). Las escuelas participantes se seleccionaron al azar a partir de un censo de escuelas preescolares pertenecientes al Programa de Desayunos Escolares.

Criterios de inclusión: tener entre 3 y 6 años, sin enfermedad respiratoria, fiebre, diarrea o deshidratación; haber efectuado todas las mediciones y contar con el consentimiento informado y firmado por los padres o representantes legales que aceptaron participar (se detalló claramente el objetivo del estudio y los beneficios y riesgos inherentes de la participación de sus hijos en concordancia con lo expuesto y aprobado por el comité de ética interinstitucional de CIAD, AC). Los niños que no cumplieron con estos criterios se excluyeron del estudio.

\section{Antropometría, grasa corporal y presión sanguínea}

El peso corporal y la talla se midieron conforme al procedimiento descrito por Jeliffe y Jeliffe. ${ }^{13}$ Con el programa Anthro, versión 3.22, de la Organización Mundial de la Salud, se calculó el puntaje $z$ del IMC para la edad (z-IMC/E). ${ }^{14}$ La circunferencia de cintura se midió con una cinta métrica de fibra de vidrio (Lafayette Instrument, USA) considerando el punto medio entre la última costilla y la cresta iliaca. Se consideró obesidad central al valor $\geq$ del percentil 90, según sexo y edad, para población méxico-americana, según Fernández y su grupo. ${ }^{15}$ La grasa corporal se midió por bioimpedancia eléctrica, con un equipo IMP5 ${ }^{\mathrm{TM}}$ (Impedimed IMP5 ${ }^{\mathrm{TM}}$ Impedimed,
Pty LTd, Aus). La masa grasa corporal se calculó mediante la ecuación generada por Ramírez y sus coautores. ${ }^{16}$ La presión arterial se midió con un baumanómetro de mercurio (Desk Model Mercurial Spyngomanometer, Model 100, USA) siguiendo los criterios del Programa Nacional de Educación en Hipertensión (NHBPEP por sus siglas en inglés) para niños y adolescentes. ${ }^{17}$

\section{Lípidos plasmáticos, glucosa e insulina}

Después de 12 horas de ayuno se extrajeron 5 $\mathrm{mL}$ de sangre de la vena antecubital. El plasma se separó de las células rojas por centrifugación a 2400 x g. El colesterol total y los triglicéridos se midieron con equipos comerciales CHODPAP (Roche Diagnostics) y GPO-PAP (Roche Diagnostics), respectivamente. El colesterol en la lipoproteína de alta densidad (HDL-c por sus siglas en inglés) se determinó en el sobrenadante después de la precipitación de las lipoproteínas con Apo B (Roche Diagnostics); las lipoproteínas de baja densidad (LDL-C) se calcularon con la ecuación descrita por Friedewald y colaboradores. ${ }^{18}$ Para clasificar los valores del perfil de lípidos se utilizaron los percentiles y puntos de corte de Daniels y Greer. ${ }^{19}$ La glucosa en ayuno se midió por el método de glucosa oxidasa GOD-PAP (Roche Diagnostics), un valor mayor a $100 \mathrm{mg} / \mathrm{dL}$ se consideró alterada en ayuno. ${ }^{20}$ La insulina se determinó por inmunoensayo tipo sándwich (ELISA)* con un juego de reactivos comercial (ALPO Diagnostics, NH, USA), se emplearon los valores de referencia propuestos por García-Cuartero y su grupo. ${ }^{21}$

Para mostrar las características de la población se verificó la normalidad de los datos (19 variables cuantitativas mediante las pruebas de Kolmogorov-Smirnov, W de Shapiro-Wilks, Anderson-Darling, D'Agostino) y se realizó esta- 
dística descriptiva, agrupada por zona urbana y conurbada. Los datos se presentan como media \pm desviación estándar y como mediana y rangos intercuartílicos, respectivamente. Para probar diferencias entre grupos se utilizó t de Student para muestras independientes. Para variables no normales se empleó la prueba $U$ de Mann Whitney. Para probar diferencias por contenido de grasa corporal (normal, moderado y elevado) se utilizó un análisis de varianza (ANOVA). En ambos análisis se consideraron diferencias significativas con una $p \leq 0.05$. Los datos se analizaron con el programa estadístico NCSS 2007 (Number Cruncher Statistical System for Windows, Kaysville, Utah, USA).

\section{RESULTADOS}

Se evaluaron 82 preescolares de 3 a 6 años, de dos zonas geográficas distintas de la ciudad de Hermosillo, Sonora; 34 pertenecientes a distintas colonias de la zona urbana (13 niñas y 21 niños) y 48 de la zona conurbada: incluyendo Poblado Miguel Alemán y la comunidad de El Tazajal (25 niñas y 23 niños).

En el Cuadro 1 se muestran los datos obtenidos de crecimiento físico y perfil metabólico, según la zona de estudio. Los preescolares de la zona urbana tuvieron concentraciones significativamente más elevadas de colesterol total, glucosa,

Cuadro 1. Características físicas, composición corporal y variables bioquímico-clínicas de niños preescolares de la zona urbana y zona conurbada de la ciudad de Hermosillo, Sonora, México

\begin{tabular}{|c|c|c|c|c|}
\hline Parámetro & Total $(\mathbf{n}=\mathbf{8 2})$ & $\mathrm{ZU}(\mathrm{n}=34)$ & $\mathrm{ZCU}(\mathrm{n}=48)$ & $\mathbf{P}^{1}$ \\
\hline $\operatorname{Edad}^{* *}$ (años) & $5.07(4.6-5.2)$ & $4.9(4.0-5.2)$ & $5.1(4.7-5.4)$ & 0.05 \\
\hline Peso** $^{* *}(\mathrm{~kg})$ & $16.7(15.6-18.6)$ & $16.4(15.7-18.6)$ & $16.8(15.2-18.9)$ & 0.75 \\
\hline Talla* (cm) & $105.5 \pm 5.0(93.9-118.6)$ & $105.5 \pm 5.2(97-118.6)$ & $105.6 \pm 5.0(93.9-116.6)$ & 0.93 \\
\hline Z-P/E* & $-0.4 \pm 0.9(-2.4-2.2)$ & $-0.3 \pm 0.8(-2.4-1.8)$ & $-0.5 \pm 1(-2.4-2.2)$ & 0.28 \\
\hline Z-T/E* & $-0.7 \pm 1(-3.0-2.1)$ & $-0.5 \pm 0.9(2.6-2.1)$ & $-0.9 \pm 1.0(3.0-1.0)$ & 0.07 \\
\hline Z-IMC & $-1.6 \pm 0.8(-1.7-2.6)$ & $-0.0 \pm 0.7(-1.7-1.6)$ & $0.0 \pm 0.9(-1.5-2.6)$ & 0.81 \\
\hline Circunferencia de cintura** & $50.15(48.1-52.8)$ & $49.1(47.4-51.2)$ & $51.1(46.9-53.4)$ & 0.03 \\
\hline$\%$ Grasa (BIE)* & $22.2 \pm 5.9(7.1-41.1)$ & $21.6 \pm 6.37(7.1 \pm 34.8)$ & $22.6 \pm 5.5(11.7-41.1)$ & 0.45 \\
\hline PAS** & $80(70-87.5)$ & $80(72.5-90)$ & $80(70-84.3)$ & 0.18 \\
\hline PAD $^{* *}$ & $50(41.8-57.5)$ & $51.2(42.5-60)$ & $50(40-55)$ & 0.75 \\
\hline $\mathrm{TG}(\mathrm{mg} / \mathrm{dL})^{* *}$ & $86.7(44-189)$ & $85.4(44-189)$ & $87.2(49.2-187.4)$ & 0.41 \\
\hline $\mathrm{CT}(\mathrm{mg} / \mathrm{dL})^{*}$ & $114.3 \pm 26.5(64.4-196.1)$ & $121.6 \pm 26.6(65.4-196.1)$ & $109 \pm 25.25(64.4-169.2)$ & 0.05 \\
\hline LDL-c (mg/dL)* & $58.2 \pm 25.2(139-127.8)$ & $62.1 \pm 26(18.6-127.8)$ & $55.5 \pm 24.5(13.9-108.4)$ & 0.24 \\
\hline HDL-c $(\mathrm{mg} / \mathrm{dL})^{* *}$ & $37.8(17.2-89.1)$ & $40.93(30.3-53.4)$ & $35.76(17.2-89.1)$ & $<0.01$ \\
\hline Glucosa $(\mathrm{mg} / \mathrm{dL})^{*}$ & $82.3 \pm 14.0(49-123)$ & $87.9 \pm 15.3(67-123)$ & $78.4 \pm 11.2(49-103)$ & $<0.01$ \\
\hline Insulina, $\mu \mathrm{UI} / \mathrm{L}(\mathrm{n}=63)$ & $2.45(0.07-28.7)$ & $3.5(0.05-28.7)$ & $1.87(0.01-18.1)$ & $<0.01$ \\
\hline $\operatorname{HOMA}^{* *}(n=63)$ & $0.49(0.01-4.7)$ & $0.75(0.06-4.7$ & $0.36(0.01-2.1)$ & $<0.01$ \\
\hline
\end{tabular}

*Media \pm desviación estándar; **mediana (intervalo intercuartilar [25-75]). Abreviaturas: ZU: zona urbana, ZCU: zona conurbada, \% grasa (BIE): porcentaje de grasa por bioimpedancia eléctrica, CT (mg/dL): colesterol total, TG (mg/dL): triglicéridos, HDL-c $(\mathrm{mg} / \mathrm{dL})$ : colesterol de lipoproteínas de alta densidad, LDL-c $(\mathrm{mg} / \mathrm{dL})$ : colesterol de lipoproteínas de baja densidad, PAS $(\mathrm{mmHg})$ : presión arterial sistólica, PAD $(\mathrm{mmHg})$ : presión arterial diastólica. ${ }^{1}$ Diferencia entre grupos probada por la prueba $t$ de Student para dos muestras independientes y para datos no normales se utilizó $U$ de Mann-Whitney $(p<0.05)$. 
insulina y HOMA $(p<0.05)$. Por el contrario, la edad promedio y la circunferencia de cintura fueron mayores en los preescolares de la zona conurbada $(p<0.05)$.

\section{Alteraciones en la composición corporal y marcadores clínicos}

En ambas zonas de estudio y grupos de edad se observaron problemas de retraso en el crecimiento (niños de 3-5 años de la zona urbana = $5.8 \%$; zona conurbada $=5.2 \%$ ), (niños mayores de 5 años de la zona conurbada $=17.2 \%$ ). Solo se encontró un caso de desmedro en un menor de 5 años, en la zona conurbada.

Aunado a lo anterior existe "riesgo de sobrepeso", "sobrepeso", y circunferencia de cintura elevada (Cuadro 2). En la zona conurbada hubo mayor proporción de niños menores de 5 años con sobrepeso y circunferencia de cintura elevada (percentil $\geq 90$; Fernández y su grupo $)^{15}$ y un porcentaje de grasa corporal elevado en los niños mayores de 5 años. ${ }^{22}$

En los marcadores clínicos (Cuadro 2), aproximadamente, 30\% de los niños tuvieron triglicéridos elevados, por encima del percentil 95 (85mg/ dL). ${ }^{19}$ Por el contrario, las HDL-c fueron bajas (38-43 mg/dL), sobre todo en la zona conurbada, en donde hubo mayor proporción de niños con concentraciones muy bajas $(\leq 5$ percentil $=38 \mathrm{mg} / \mathrm{dL}$ ).

De manera más marcada, en la zona urbana se encontraron alteraciones de glucosa e insulina con casos de resistencia a la insulina. La medición de la presión arterial mostró que en ambas

Cuadro 2. Alteraciones en la composición corporal y marcadores bioquímicos en preescolares por zona de estudio

\begin{tabular}{|c|c|c|c|c|c|}
\hline \multirow[b]{2}{*}{ Clasificación } & \multirow[b]{2}{*}{ Valor de Referencia } & \multicolumn{2}{|c|}{ 3-5 años } & \multicolumn{2}{|c|}{$\geq 5$ años } \\
\hline & & ZU (n=19) & ZCU $(n=17)$ & $\mathrm{ZU}(\mathrm{n}=29)$ & ZCU $(n=17)$ \\
\hline Sobrepeso $^{\Delta}$ & $\mathrm{IMC} / \mathrm{E}>1 \mathrm{DE}$ & ND & $10.5 \%$ & $11.7 \%$ & $7.1 \%$ \\
\hline CC Elevada* & $>90$ percentil & $11.7 \%$ & $21 \%$ & $5.8 \%$ & $6.8 \%$ \\
\hline \multirow[t]{2}{*}{ Grasa corporal elevada** } & $\begin{array}{l}>26 \% \text { niños } \\
>34 \% \text { niñas }\end{array}$ & ND & ND & $5.8 \%$ & $6.8 \%$ \\
\hline & & $Z U(n=34)$ & $\mathrm{ZCU}(\mathrm{n}=48)$ & & \\
\hline Triglicéridos elevados ${ }^{*}$ & $>85 \mathrm{mg} / \mathrm{dL}$ & $30 \%$ & $35 \%$ & & \\
\hline Colesterol total elevado & $>200 \mathrm{mg} / \mathrm{dL}$ & $3 \%$ & ND & & \\
\hline LDL-c elevado & $110-129 \mathrm{mg} / \mathrm{dL}$ & $6 \%$ & ND & & \\
\hline HDL-c muy bajo & $38 \mathrm{mg} / \mathrm{dL}(\leq 5$ percentil) & $6 \%$ & $25 \%$ & & \\
\hline Glucosa alterada $^{\delta}$ & $>100 \mathrm{mg} / \mathrm{dL}$ & $11.7 \%$ & $2 \%$ & & \\
\hline Insulina elevada ${ }^{\delta}$ & Percentil 90/edad & $20 \%$ & $2.6 \%$ & & \\
\hline $\mathrm{HOMA}^{\delta}$ & Percentil 90/edad & $16 \%$ & ND & & \\
\hline Pre-hipertensión $\mathrm{AD}^{\theta}$ & Percentil 90-94 & $41 \%$ & $27 \%$ & & \\
\hline Pre-hipertensión $\mathrm{AS}^{\theta}$ & Percentil 90-94 & ND & $6 \%$ & & \\
\hline Presión arterial diastólica elevada ${ }^{\theta}$ & $>$ percentil 99 & ND & $4 \%$ & & \\
\hline Presión arterial sistólica elevada ${ }^{\theta}$ & $>$ percentil 99 & ND & $2 \%$ & & \\
\hline
\end{tabular}


zonas hay niños con presión arterial diastólica normal elevada, condición clínica denominada pre-hipertensión arterial. ${ }^{17}$ Sólo se observaron casos conjuntos de hipertensión arterial diastólica y sistólica en la zona conurbada.

En ambas zonas hubo casos de hipoglucemia (zona urbana $=8.8 \%$; zona conurbada $=27 \%)$ y prediabetes (zona urbana $=11.8 \%$; zona conurbada $=2 \%$ ). Asimismo, del total de preescolares estudiados ( $n=82), 9.5 \%$ tuvo valores elevados de insulina ( $\geq$ percentil $90=10.63 \mu \mathrm{UI} / \mathrm{mL}$ ) observándose que esta condición estuvo en mayor proporción en la zona urbana (20 vs 2.6\%) ( $p$ $<0.01$ ). Se encontró resistencia a la insulina en $6.3 \%$ del total de la población estudiada. ${ }^{21}$

Un análisis de comparación, exploratorio, alcanzó a apreciar algunas diferencias significativas entre sexos. Las niñas de la zona urbana tuvieron mayor porcentaje de grasa corporal $(24 \% \pm 6.7$ vs $20 \% \pm 5.6)(p \leq 0.05)$ y mayor concentración de triglicéridos (95.8 vs $71.9 \mathrm{mg} / \mathrm{dL}$ ) $(p<0.01)$ que los niños. En la zona conurbada las niñas tuvieron valores de insulina y HOMA superiores a los niños $(p<0.05)$. Un análisis de asociación entre las variables estudiadas mostró que existe una correlación entre el porcentaje de grasa corporal y la circunferencia de cintura en ambos grupos de estudio (zona urbana $r=0.71$; $p<0.01$; zona conurbada $r=0.64 ; p<0.01$ ).

La mayoría de los niños mayores de 5 años tuvo un contenido de grasa corporal normal (zona urbana $=64.7 \%$; zona conurbada $=72.4 \%)(p<0.01)$; no obstante, también se encontraron casos con grasa moderada (zona urbana $=29.4 \%$; zona conurbada $=17.2 \%$ ) y elevada $($ zona urbana $=5.8 \%$; zona conurbada $=10.3 \%$ ). ${ }^{22}$ Tomando como referencia la clasificación de grasa corporal, ${ }^{22}$ se evaluaron posibles cambios en los biomarcadores evaluados. Los niños con porcentaje de grasa moderado y elevado tienen tendencia a mayor presión arterial sistólica y diastólica, de glucosa, colesterol total, LDL-c y triglicéridos.

\section{DISCUSIÓN}

Este estudio se llevó a cabo en la ciudad de Hermosillo, Sonora, en niños de edad preescolar habitantes de áreas urbanas y suburbanas. El análisis global de crecimiento físico considerando los indicadores de P/E y T/E observa que, aproximadamente, $10 \%$ de los niños preescolares evaluados de 3 a 5 años y $6.8 \%$ de los niños mayores de 5 años tienen afectado el crecimiento. En este sentido, la $\mathrm{OMS}^{23}$ señala que la desnutrición durante los primeros años de vida puede incrementar el riesgo de enfermedades crónicas no trasmisibles en la vida adulta.

Simultáneamente se detectaron problemas de retraso en el crecimiento y se observaron niños en riesgo de sobrepeso (zona conurbada $=10.5 \%$; zona urbana $=$ ningún caso) y con sobrepeso (zona conurbada $=15.7 \%$; zona urbana $5.8 \%$ ). De Onis y colaboradores, ${ }^{24}$ en su análisis de la problemática de sobrepeso y obesidad en niños preescolares a nivel mundial, señalan una prevalencia de riesgo de sobrepeso de 14.4 y $13.6 \%$ para los países en desarrollo. Las cifras de riesgo de sobrepeso observadas en esta investigación se sitúan $1.5 \%$ por encima de lo reportado en el ámbito mundial. Nuestros hallazgos destacan, también, que la zona conurbada de la ciudad de Hermosillo es la más afectada con problemas de sobrepeso y llama la atención que en ninguna de las zonas de estudio se encontraron casos de obesidad ( $>3$ DE). El informe nacional para este grupo de edad reporta prevalencias combinadas de sobrepeso más obesidad de $14.1 \%$ en el área urbana y de $8.7 \%$ para el área rural. Nuestros datos de sobrepeso resultan muy cercanos a este informe; sin embargo, no es posible la comparación porque la referencia es prevalencia combinada. 
Una proporción elevada de niños de ambos grupos de edad y de zona de estudio tuvieron valores de circunferencia de cintura por encima del percentil 90. En los niños mayores de 5 años se encontró una concordancia entre la proporción de niños con circunferencia de cintura elevada y la de niños con porcentaje de grasa corporal elevado.22 Considerando las limitaciones que pueden existir porque no hay referencias percentilares para nuestra población, es posible suponer que una parte de la grasa corporal en estos niños se acumula en torno de las víseras. EI exceso de grasa acumulado antes de la pubertad se asocia con aumento de las concentraciones de lípidos sanguíneos y, con ello, del riesgo de enfermedades crónico-degenerativas. ${ }^{25}$

Los resultados obtenidos de los indicadores de crecimiento señalan a una población preescolar en la que coexisten la desnutrición y el sobrepeso, sobre todo en la zona conurbada; queda de manifiesto la doble carga de la malnutrición. Está demostrado que la malnutrición en la infancia es un marcador de enfermedades crónicas prematuras en la vida adulta, como la diabetes y las enfermedades cardiovasculares. ${ }^{26}$ Es importante considerar que en esta etapa de la vida el sobrepeso puede ser un factor favorecedor de aterosclerosis que se manifestará en enfermedades cardiovasculares en la vida adulta.

De acuerdo con las recomendaciones pediátricas para el perfil de lípidos sugerido por Daniel y Greer, ${ }^{19}$ este estudio determinó que la población preescolar evaluada tiene triglicéridos plasmáticos elevados (percentil $95=85 \mathrm{mg} / \mathrm{dL}$ ) y una concentración baja de HDL-c (percentil $5=38$ $\mathrm{mg} / \mathrm{dL}$ ). Ambos indicadores se consideran factores de riesgo cardiovascular independiente, además de formar parte del síndrome metabólico. Los lípidos sanguíneos se ven influidos por la genética, la dieta y la actividad física. Este estudio no evaluó ninguna de estas variables por lo que no puede explicarse la contribución que cada uno de estos factores está teniendo en las alteraciones encontradas. Los estudios epidemiológicos indican que si durante la niñez se alteran las concentraciones de lípidos sanguíneos eventualmente se tendrán lesiones ateroscleróticas en la edad adulta. ${ }^{9}$

La partícula de HDL posee propiedades antioxidantes (enzima PON1) que evitan la oxidación de la partícula LDL y, con ello, la aterosclerosis. ${ }^{27}$ En esta investigación 25\% de los niños de las localidades conurbadas y $5.8 \%$ de la parte urbana tuvieron valores por debajo del percentil 5 de HDL-c, condición referida como hipoalfalipoproteinemia. Es importante destacar también, que la mayoría de los niños se situó por debajo del percentil $10=43 \mathrm{mg} / \mathrm{dL}$ (zona conurbada $=54 \%$; zona urbana $=50 \%$ ) lo que sugiere que este grupo de población puede tener una protección antioxidante de LDL-c muy baja y, también, poca capacidad para catabolizar el colesterol almacenado, lo que pudiera dar paso al inicio de la formación de estrías grasas en una etapa temprana de la niñez.

El patrón clínico de triglicéridos elevados y HDLc bajo $(86.7 \mathrm{mg} / \mathrm{dL}$ y $37.8 \mathrm{mg} / \mathrm{dL}$ ) concuerda con el reportado por Reaven y sus coautores ${ }^{28}$ para población escolar méxico-americana (84.6 y $47 \mathrm{mg} / \mathrm{dL}$ ). Así, también, con estudios previos efectuados en esta misma región, pero en escolares de 6-12 años en quienes se observó el mismo patrón de alteraciones, Ramírez ${ }^{11}$ encontró un patrón lipídico semejante en escolares, determinado por el síndrome metabólico en la zona rural $(5 \%)$ y urbana $(7.4 \%)$.

Los hallazgos de este ensayo se tornan importantes porque se logra determinar que en la edad preescolar pueden observarse alteraciones en los lípidos sanguíneos que anteriormente se habían reportado en escolares y en adultos de esta misma región. Las alteraciones a esta edad 
hacen suponer, también, que hay un aspecto genético involucrado. ${ }^{29}$

Otro factor de riesgo importante para enfermedades cardiovasculares es la hipertensión arterial que, a su vez, se asocia con otros factores: sobrepeso, obesidad, inactividad física y concentraciones elevadas de lípidos sanguíneos. ${ }^{2}$ En este estudio, solo en la zona conurbada se encontraron niños con hipertensión diastólica (4\%) y sistólica (2\%); sin embargo, de acuerdo con lo estipulado por la NHBPEP ${ }^{17}$ una gran proporción de preescolares evaluados ya tiene pre-hipertensión diastólica. Un estudio previo de nuestro grupo reportó $12 \%$ de prevalencia de hipertensión en escolares de esta misma zona. Las proporciones encontradas en los niños preescolares de este estudio son menores, pero resultan importantes porque el problema comienza a detectarse a una edad aún más temprana.

Las concentraciones de glucosa e insulina en ayuno, así como el valor de HOMA fueron significativamente mayores en la zona urbana $(\mathrm{p}<$ 0.01). Los niños con glucosa alterada tuvieron un valor promedio de $116 \mathrm{mg} / \mathrm{dL}$ y el valor más alto observado fue de $123 \mathrm{mg} / \mathrm{dL}$ en la zona urbana, que se envió para atención médica para su posible diagnóstico de diabetes. La elevación de la insulina observada en los niños (zona urbana $=20 \%$; zona conurbada $=2.6 \%$ ) señala una posible compensación metabólica para tratar de normalizar las concentraciones de glucosa. ${ }^{30}$

Según el criterio diagnóstico de García y su grupo, ${ }^{21}$ en la zona urbana los niños ya tienen resistencia a la insulina con valores de HOMA superiores al percentil 90. Tanto la insulina como la resistencia a la misma coinciden con los hallazgos de glucosa alterada, que indican posibles afectaciones en el metabolismo de la glucosa que pudieran desencadenar en diabetes tipo 2 a una edad más temprana. ${ }^{20}$ Relacionada con la hiperinsulinemia se encuentra la acantosis nigricans, que en este estudio también se exploró la búsqueda de ésta en el cuello de los niños y no se observó ningún caso. Conforme fue posible se registraron datos de dos variables adicionales: peso al nacer y tipo de nacimiento (cesárea o parto) (zona conurbada $n=37$; zona urbana $n$ =29). Del total de registros obtenidos para cada caso, el promedio de peso al nacer fue de: zona conurbada $=3417 \pm 568 \mathrm{~g}$; zona urbana $=3259$ $\pm 579 \mathrm{~g}(\mathrm{p}=0.2)$. En la zona conurbada $21 \%$ de los niños fueron macrosómicos (peso al nacer mayor de $4 \mathrm{~kg}$ ) y $78 \%$ nacieron por parto, sin complicaciones. En cambio, en la zona urbana $3.6 \%$ fueron macrosómicos y $58 \%$ nacieron por parto sin complicaciones.

Los resultados obtenidos de esta investigación ponen de manifiesto la doble carga de la malnutrición en la que coexisten problemas de desnutrición y sobrepeso, y porcentajes de grasa corporal elevados. Se confirma que los factores de riesgo cardiovascular y de diabetes tipo 2 también coexisten en este grupo de edad. La zona conurbada tuvo mayor porcentaje de niños con alteraciones en los lípidos, triglicéridos elevados (35\%), HDL-c bajo (25\%), y prehipertensión arterial. En la zona urbana se registró mayor porcentaje de niños con glucosa alterada $(12 \%)$ y concentraciones elevadas de insulina y resistencia a ésta.

El estudio de la dieta efectuado por Ayala-Mendivil $^{31}$ en escolares de esta misma región mostró que su dieta es alta en grasa ( $>30 \%$ ), de la que $37.6 \%$ proviene de grasas saturadas y $5.4 \%$ de grasas trans; asimismo, del total de carbohidratos consumidos, 39\% proviene de azúcares simples. Estos resultados hacen pensar que los preescolares pudieran tener un patrón dietario similar que, en parte, daría respuesta a los indicadores clínicos observados en este estudio.

Es posible que otras variables no evaluadas en este estudio, como: la actividad física y la herencia, estén jugando un papel importante en la aparición de esas alteraciones clínicas 
que, además, son componentes del síndrome metabólico. ${ }^{32}$ En el caso de los niños de la zona conurbada, al parecer, el peso al nacer señala que quizá haya una descompensación metabólica desde el embarazo. Además del reducido tamaño de muestra no se dispuso de información suficiente para poder probarlo porque no se obtuvieron datos acerca de la dieta de la madre, ni del aumento de peso durante el embarazo, ni del diagnóstico de diabetes gestacional, aspectos que quedan como tarea pendiente.

\section{CONCLUSIONES}

Los hallazgos de este estudio muestran que los factores de riesgo cardiovascular y de diabetes tipo 2 prevalecen en niños de 3 a 6 años habitantes de las zonas urbana y conurbada de la ciudad de Hermosillo, en el Noroeste de México.

\section{Fortalezas y debilidades del estudio}

Reconocemos que una de las limitaciones de este estudio reside en el hecho de que se tiene un tamaño reducido de muestra, que no permite extrapolar la información hacia el resto de la población preescolar de la entidad. Sin embargo, se logra mostrar que, efectivamente, hay alteraciones en los indicadores clínicos de enfermedades cardiovasculares y diabetes en población prescolar, observando diferencias significativas entre los grupos evaluados. El registro obtenido del peso al nacer pareciera señalar un camino hacia una posible respuesta del porqué de las alteraciones a tan temprana edad, sin embargo, hace falta más información para poder probarlo.

\section{Agradecimientos}

A los niños, a sus padres o tutores y a los maestros por su amable participación en este estudio, así como a los directivos y maestros de las escuelas participantes mediante la Secretaría de Educación y Cultura del Estado de Sonora.

\section{REFERENCIAS}

1. Van Vliet $M$, Heymans $M W$, von Rosenstiel IA, Brandjes DPM, Beijnen JH, Diamant M. Cardiometabolic risk variables in overweight and obese children: a worldwide comparison. Cardiovasc Diabetol. 2011;10:106. (https://cardiab. biomedcentral.com/articles/10.1186/1475-2840-10-106).

2. Getz GS, Reardon CA. Nutrition and cardiovascular disease. Arterioscler Thromb Vasc Biol. 2007;27:2499-506. (http://atvb.ahajournals.org/content/27/12/2499)

3. Johannsson E, Arngrimsson SA, Thorsdottir I, Sveinsson T. Tracking of overweight from early childhood to adolescence in cohorts born 1988 and 1994: overweight in a high birth weight population. Int J Obes (Lond). 2006;30:126571. (https://www.nature.com/articles/0803253)

4. Timpka $T$, Angbratt $M$, Bolme $P$, Hermansson $G$, Häger A, Valter L. A high-precision protocol for identification of preschool children at risk for persisting obesity. PLoS ONE. 2007;2:e535. (http://journals.plos.org/plosone/ article?id=10.1371/journal.pone.0000535).

5. Juonala M, Magnussen CG, Berenson GS, Venn A, Burns TL, Sabin MA, et al. Childhood adiposity, adult adiposity, and cardiovascular risk factors. N Engl J Med. 2011;365:1876-85. ( http://www.nejm.org/doi/ full/10.1056/NEJMoa1010112\#t=article) .

6. Tamir I, Heiss G, Glueck CJ, Christensen B, Kwiterovich $P$, Rifkind BM. Lipid and lipoprotein distributions in white children ages 6-19 yr. The Lipid Research Clinics Program Prevalence Study. Int J Chronic Dis. 1981;34:2739. (http://www.sciencedirect.com/science/article/ pii/0021968181900795).

7. Newman III WP, Freedman DS, Voors AW, Gard PD, Srinivasan SR, Cresanta JL, et al. Relation of serum lipoprotein levels and systolic blood pressure to early atherosclerosis. N Engl J Med. 1986;314:138-44. (http://www.nejm.org/ doi/full/10.1056/nejm198601163140302)

8. Sanchez-Bayle M, Gonzalez-Requejo A, Ruiz-Jarabo C, Asensio J, Baeza J, Vila S, et al. Serum lipids and apolipoproteins in Spanish children and adolescents: a 5 year follow-up. Acta Paediatrica. 1996;85:292-4. http://onlinelibrary.wiley. com/doi/10.1111/j.1651-2227.1996.tb14018.x/full

9. Velásquez E, Barón MA, Solano L, Páez M, Llovera D, Portillo Z. Perfil lipídico en preescolares venezolanos según nivel socioeconómico. Arch Latinoam Nutr. 2006;56:228. (http://www.scielo.org.ve/scielo.php?pid=S000406222006000100004\&script=sci_arttext).

10. Acosta García E. Obesidad, tejido adiposo y resistencia a la insulina. Acta Bioquím Clín Latinoam. 2012;46:183-94. (http://www.scielo.org.ar/scielo.php?script=sci_arttext\& pid=S0325-29572012000200003).

11. Ramírez-Murillo C, Guillot-Sánchez E, Elizabeth ArtalejoOchoa QB, Robles-Sardin AE, Ponce-Martínez JA, CaireJuvera G, et al. Prevalence of Metabolic Syndrome Diag- 
nosed by Three Different Criteria in School-Aged Children from Rural and Urban Areas of Northwest Mexico. J Metab Syndr. 2015;4:2-9. (https://www.researchgate.net/profile/ Martha_Nydia_Ballesteros/publication/282980088_Prevalence_of_Metabolic_Syndrome_Diagnosed_by_ Three_Different_Criteria_in_School-Aged_Children_ from_Rural_and_Urban_Areas_of_Northwest_Mexico/ links/56254e9f08ae4d9e5c4bb26a.pdf).

12. Gutiérrez JP, Rivera-Dommarco J, Shamah-Levy T, Villalpando-Hernández S, Franco A C-NL, Romero-Martínez M, et al. Encuesta Nacional de Salud y Nutrición 2012. Resultados Nacionales. Cuernavaca, México: Instituto Nacional de Salud Pública; 2012. (http://ensanut.insp.mx/informes/ ENSANUT2012ResultadosNacionales.pdf).

13. Jelliffe TDB, Jelliffe EFP. Community Nutritional Assessment: With Special Reference to Less Technically Developed Countries. Oxford University Press; 1989. (https:// www.popline.org/node/372046).

14. WHO. Anthro para computadoras personales, versión 3, 2009: Software para evaluar el crecimiento y desarrollo de los niños del mundo. Ginebra, OMS 2009 (http://www. OMS.int/childgrowth/software/en/ ). Geneva: World Health Organization; 2009.

15. Fernández JR, Redden DT, Pietrobelli A, Allison DB. Waist circumference percentiles in nationally representative samples of African-American, European-American, and Mexican-American children and adolescents. J Pediatr. 2004;145:439-44. (http://www.sciencedirect.com/science/article/pii/S0022347604005530).

16. Ramírez-López E, Grijalva-Haro MI, Valencia ME, Ponce $\mathrm{JA}$, Artalejo E. Impacto de un programa de desayunos escolares en la prevalencia de obesidad y factores de riesgo cardiovascular en niños sonorenses. Salud Publica Mex. 2005;47(2):126-33. (http://www.scielo.org.mx/scielo. php?script=sci_arttext\&pid=S0036-36342005000200006).

17. NHBPEP. National High Blood Pressure Education Program Working Group on High Blood Pressure in Children and Adolescents. The fourth report on the diagnosis, evaluation, and treatment of high blood pressure in children and adolescents. Bethesda, MD: National Institutes of Health; 2005. (http://pediatrics.aappublications.org/content/114/ Supplement_2/555).

18. Friedewald WT, Levy RI, Fredrickson DS. Estimation of the Concentration of Low-Density Lipoprotein Cholesterol in Plasma, Without Use of the Preparative Ultracentrifuge. Clin Chem. 1972;18:499-502. (http://clinchem.aaccjnls. org/content/18/6/499.short)

19. Daniels SR, Greer F, Committee on Nutrition. Lipid screening and cardiovascular health in childhood. Pediatrics. 2008;122(1):198-208. (http://pediatrics.aappublications. org/content/122/1/198?viewType=Print\&viewClass=Print).

20. ADA. 2. Classification and Diagnosis of Diabetes. Diabetes Care. 2016;39:S13-S22. (http://care.diabetesjournals.org/ content/39/Supplement_1/S13).
21. García-Cuartero B, García-Lacalle C, Jímenez-Lobo C, González-Vergaz A, Calvo-Rey C, Alcázar-Villar M, et al. Índice HOMA y QUICKI, insulina y péptido $C$ en niños sanos. Puntos de corte de riesgo cardiovascular. An Pediatr (Barc.). 2007;66(5):481-90. (http://www.analesdepediatria.org/es/ndice-homa-quicki-insulina-peptido/ articulo/13102513/)

22. Freedman D, Wang J, Thornton J, Mei Z, Sopher A, Pierson $R$, et al. Classification of body fatness by body mass indexfor-age categories among children. Arch Pediatr Adolesc Med. 2009;163(9):805-11. (https://www.ncbi.nlm.nih. gov/pmc/articles/PMC2846460/).

23. WHO. Cardiovascular Diseases (CVD) Prensa: Notas descriptivas. Geneva: OMS; 2016. (http://www.who.int/ topics/cardiovascular_diseases/es/).

24. De Onis M, Blössner M, Borghi E. Global prevalence and trends of overweight and obesity among preschool children. Am J Clin Nutr. 2010;92:1257-64. (http://ajcn. nutrition.org/content/92/5/1257.short).

25. Chiarelli F, Marcovecchio ML. Insulin resistance and obesity in childhood. Eur. J. Endocrinol. 2008;159:S67-S74. (http:// www.eje-online.org/content/159/suppl_1/S67.short).

26. Barker DJP. Fetal nutrition and cardiovascular disease in later life. Br. Med. Bull. 1997;53:96-108. (https://academic. oup.com/bmb/article/53/1/96/285492).

27. Mackness B., Mackness M. (2010) Anti-Inflammatory Properties of Paraoxonase-1 in Atherosclerosis. In: Reddy S. (eds) Paraoxonases in Inflammation, Infection, and Toxicology. Advances in Experimental Medicine and Biology, vol 660. Humana Press. (https://link.springer. com/chapter/10.1007/978-1-60761-350-3_13\#citeas).

28. Reaven P, Nader PR, Berry C, Hoy T. Cardiovascular disease insulin risk in Mexican-American and Anglo-American children and mothers. Pediatrics. 1998;101:e12-e. (http:// pediatrics.aappublications.org/content/101/4/e12.short).

29. Kelishadi R, Asgary S, Bashardoust N, Naderi GA, Zadegan NS. Atherosclerosis risk factors in children and adolescents with or without familyhistory of premature coronary artery disease. Med Sci Monitor. 2002;8:CR425-CR9. (https://www. medscimonit.com/abstract/index/idArt/420814/act/2).

30. Barja S, Arteaga A, Acosta A. Resistencia insulínica y otras expresiones del síndrome metabólico en niños obesos chilenos. Rev Med Chil. 2003;131(3):25968. (http://www.scielo.cl/scielo.php?pid=S0034$98872003000300003 \&$ script=sci_arttext\&tlng=pt).

31. Ayala-Mendivil A. Presencia de hígado graso no alcohólico en ninos escolares sonorenses, asociada al consumo de azúcares simples y grasa Nutrición Humana. Hermosillo, Sonora: Centro de Investigación en Alimentación y Desarrollo, A.C.; 2016

32. Zimmet P, Alberti G, Kaufman F, Tajima N, Silink M, Arslanian $S$, et al. The metabolic syndrome in children and adolescents: the IDF consensus. Diabetes Voice. 2007;52:2932. (http://onlinelibrary.wiley.com/doi/10.1111/j.13995448.2007.00271.x/full). 